\title{
E-cigarettes: no smoke without fire?
}

\author{
N. Daniels ${ }^{\star}$, C. Cosma, A. Llewellyn, D. Banks, H. Morris, J. Copeland and E. Djarlijeva \\ Presented to the Institute \& Faculty of Actuaries, 24 February 2020, Edinburgh \\ ${ }^{*}$ Correspondence to: Niel Daniels, Institute and Faculty of Actuaries, 7th Floor, Holborn Gate, 326-330 High Holborn, \\ London WC1V 7PP, UK. E-mail: niel.daniels@aul-group.com
}

\begin{abstract}
Smoking was one of the biggest preventable killers of the $20^{\text {th }}$ century, and it continues to cause the death of millions across the globe. The rapid growth of the e-cigarette market in the last 10 years and the claims that it is a safer form of smoking, and can help with smoking cessation, have led to questions being raised on their possible impact to society, the health of the population and the insurance industry. Recent media attention around the possible health implications of e-cigarette use has also ensured that this topic remains in the public eye. The e-cigarette working party was initiated by the Institute and Faculty of Actuaries' Health and Care Research Sub-Committee in July 2016, with the primary objective of understanding the impact of e-cigarettes on life and health insurance. In this paper, we have looked at all areas of e-cigarette usage and how it relates to insurance in the UK market. In particular, we have covered the potential risks and benefits of switching to e-cigarettes, the results of studies that have been published, the potential impact on underwriting and claims processes and the potential impact on pricing (based on what modelling is possible with the data available). Research in this area is still in its infancy and data are not yet mature, which makes predicting the long-term impact of e-cigarette smoking extremely challenging, for example, there are no studies that directly measure the mortality or morbidity impact of long-term e-cigarette use and so we have had to consider studies that consider more immediate health impacts or look more simply at the constituents of the output of an e-cigarette and compare them to that of a cigarette. The data issue is further compounded by the findings of studies and the advice of national health authorities often being conflicting. For example, while National Health Service England has publicly stated that it supports the growth of e-cigarette usage as an aid to reduce traditional smoking behaviour, the US Food and Drug Administration has been much more vocal in highlighting the perceived dangers of this new form of smoking. Users' behaviour also adds complexity, as dual use (using both e-cigarettes and cigarettes) is seen in a high percentage of users and relapse rates back to cigarette smoking are currently unknown. Having talked to a number of experts in the field, we have discovered that there is certainly not a common view on risk. We have heard from experts who have significant concerns but also to experts who do believe that e-cigarettes are far safer than tobacco. We have purposefully considered conflicting evidence and have consulted with various parties so we can present differing points of view, thereby ensuring a balanced, unbiased and fair picture of our findings is presented. The evidence we have reviewed does suggest that e-cigarettes are a safer alternative to traditional smoking, but not as safe as non-smoking. There are no large, peerreviewed, long-term studies yet available to understand the true impact of a switch to e-cigarette use, so currently we are unable to say where on the risk spectrum between cigarette smoking and life-time non-smoking it lies. We do not yet understand if the benefits seen in the studies completed so far will reduce the risk in the long term or whether other health risks will come to light following more prolonged use and study. This, coupled with concerns with the high proportion of dual use of cigarettes and e-cigarettes, relapse rates and the recent growth in medical problems linked with e-cigarette use, means that we need to wait for experience to emerge fully before firm conclusions can be drawn. Although we have presented a view, it is vitally important that our industry continues to monitor developments in this area and fully considers what next steps and future actions may be required to ensure our position reflects the potential benefits and risks that e-cigarette use may bring. We feel that the time is right for a body such as the IFoA to analyse the feasibility of collecting the necessary data through the Continuous Mortality Investigation that would allow us to better analyse the experience that is emerging.
\end{abstract}

This paper expresses the views of the individual authors and not necessarily those of their employers.

(c) Institute and Faculty of Actuaries 2020. This is an Open Access article, distributed under the terms of the Creative Commons Attribution licence (http://creativecommons.org/licenses/by/4.0/), which permits unrestricted re-use, distribution, and reproduction in any medium, provided the original work is properly cited. 
Keywords: E-cigarettes; Tobacco; Risk; Quit Rates; Modelling

\section{Introduction}

The ultimate aim of this paper is to present information to understand the role of e-cigarettes in society today and in the future, which could have significant ramifications not only for the protection industry, but for the population in general. The e-cigarettes working party was initiated by the Institute and Faculty of Actuaries' Health and Care Research Sub-Committee in July 2016, with the primary objective of understanding the impact of e-cigarettes on life and health insurance.

The working party comprises seven members who work across a variety of fields within the industry, and we have consulted with several other professionals in the health and research sectors on the subject. We have been tasked with considering not only the impact of e-cigarettes today, but also suggesting and evaluating what future impact they might have. This will be looked at from several industry perspectives: pricing, product design, underwriting, claims, advisers and chief medical officers (CMOs), from both the insurers' and reinsurers' viewpoints.

The scope of the work related to traditional e-cigarettes specifically, although it is acknowledged that there are other reduced risk products such as

- IQOS - Philip Morris' heat-not-burn product;

- Blu - Imperial Tobacco's e-cigarette product;

- JUUL - a popular e-cigarette in the US using nicotine salts, largely unknown in the UK; and

- SNUS - a form of powdered tobacco popular in Sweden.

E-cigarette development, research and regulation is still in its infancy; consequently, there is a lot of contradictory information in the public domain. We have evaluated a significant number of published medical studies as well as liaising with industry experts, and we will be collating, contrasting and presenting published research and findings in this paper. Many medical studies are still in early stages, and it will be many years before a true picture of the impact of e-cigarettes is known. Whilst we have looked at a number of sources of information from across the globe, our aim has only ever been to consider the UK insurance industry and as such this paper should be read in that context.

In this paper, we will discuss a number of interesting and evolving issues relating to the introduction of e-cigarettes and their impact on our industry. We have purposefully considered conflicting evidence and have consulted with various parties so we can present differing points of view, thereby ensuring a balanced, unbiased and fair picture of our findings is presented.

As industry professionals in the life and health sectors, we are familiar with the truly damaging and destructive impact that smoking has. We also understand that any successful effort to reduce or stop smoking can have a positive effect on one's health, even after many years of smoking. We therefore feel that we have a responsibility to promote healthy life choices and encourage a pathway towards a smoke-free world.

Before work could begin in earnest, it was important that working party members had a good understanding of exactly what an e-cigarette was and how it worked.

The e-cigarette was invented by Chinese pharmacist Hon Lik (Wikipedia, 2019), who patented the device in 2003 and introduced it to the Chinese market the following year. From there, its popularity has grown and its use is now worldwide.

Lighting a traditional cigarette causes the tobacco to burn, releasing smoke that contains nicotine. The user breathes in the smoke to deliver nicotine to the lungs.

An e-cigarette does not rely on this process of combustion. Instead, an e-cigarette is a batterypowered device that converts liquid nicotine into a mist, or vapour, that the user inhales. Depending on the e-cigarette, the user may simply inhale from the mouthpiece to begin the vaporisation process and deliver nicotine to the lungs, although some devices have a manual switch that activates the vaporiser inside. When the user exhales, the vapour looks much like a cloud of cigarette smoke. 


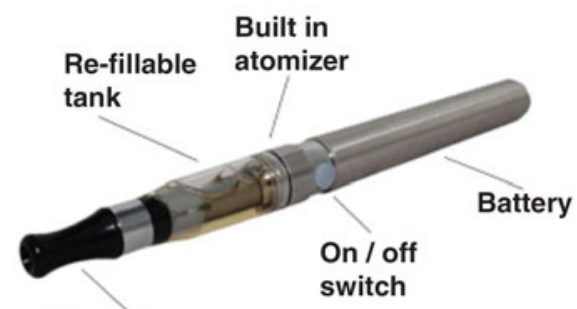

Mouthpiece

Figure 1. Anatomy of an e-cigarette.

There is no fire, ash or smoky smell. Whilst e-cigarettes do contain some of the harmful chemicals associated with smoking cigarettes, they do not contain carbon monoxide (CO) and tar.

There are a variety of e-cigarette devices (e.g. Figure 1), differing mainly by appearance and battery performance. The liquid or "smoke juice" that fills the cartridges/tank is made up of three main ingredients:

1. Nicotine, which can be of various strengths.

2. Propylene glycol, an additive approved for use in food or vegetable glycerin. These ingredients are known to be irritants and have medical side effects including allergic reactions. Propylene glycol may be more commonly known for its use in fog machines that create a smoky atmosphere at stage shows.

3. Flavourings, of which there are many, for example, cherry, menthol, peppermint, vanilla and chocolate.

A key part of our work is understanding how these ingredients potentially impact a user's health.

\section{The Core Problem}

There is a wealth of established and accepted data on the effect of smoking on health. Insurers are able to accurately price mortality and longevity risks for smokers. However, this was not always the case. Cigarettes, when they were first available, were thought to be healthy. In fact in the 1940s and 1950s, they were being promoted as "good for your health". It took many years of medical studies to demonstrate a more accurate picture.

The explosion in e-cigarette use has brought similar challenges and made it difficult for this group to come up with definitive answers. The main challenges have been

- lack of mortality data - it will be 20 to 30 years before it is available;

- lack of claims experience to demonstrate whether e-cigarettes will lead to fewer smokingrelated claims or not;

- sustainability of e-cigarette usage - the relapse rate is currently unknown due to lack of data; and

- safety of e-cigarettes - huge differences in opinion, depending on who is approached.

To try to overcome the challenges, it was felt that analysis needed to be broken down into four main streams to allow the report to be prepared.

The following areas were analysed.

\subsection{Medical Research}

- Research into the relative risk of e-cigarettes. 
- Incidence rate of cancer, respiratory disease and cardiovascular disease on e-cigarette users.

- Research into the safety of the three main e-cigarette ingredients.

- Research into the impacts of e-cigarette usage on smoking cessation rates, relapse rates and dual usage (those who smoke cigarettes and e-cigarettes).

\subsection{Social Demographics}

- Data on usage amongst different groups (age, sex, region, smoking status and socioeconomic group).

\subsection{Legislation and Regulation}

- How legislation may impact usage?

- The possible impact of changes in public policy on where people can smoke.

- The possible impact of regulation of e-cigarette devices, nicotine strength and liquid flavourings.

\subsection{Modelling}

- Modelling the impact of e-cigarettes on smoking prevalence.

- Modelling the impact of e-cigarette usage on mortality and morbidity.

\section{Health risks}

The rapid growth in the popularity of e-cigarettes over a short time period, less than a decade, means that there is a shortage of comprehensive analysis to enable us to understand the long-term effect of e-cigarettes on health. There are short-term studies that give indications about possible risks, but only time will provide the data needed to be confident about any conclusions drawn.

Observational studies and randomised control trial evidence that has been conducted to date can be broadly summarised into two categories: health risk and smoking cessation.

The health risks of e-cigarettes have been examined by looking at them through various different lenses:

- ingredients;

- toxicology;

- impact on genetic material;

- tissue damage;

- cardiovascular impact;

- respiratory function;

- cancer;

- smoking cessation.

\subsection{Ingredients}

There are approximately 600 ingredients American Lung Association (2019) in cigarettes, and when burned cigarettes create more than 7,000 chemicals American Lung Association (2019). Many are known to be toxic and at least 69 cause cancer American Lung Association (2019). Significantly, e-cigarettes do not produce tar or CO, two of the lethal ingredients in cigarette smoke. In addition, e-cigarettes contain only up to 15 ingredients American Lung Association (2019) and fewer cancer causing chemicals, so they have the potential to be less harmful. However, some of the ingredients and chemicals they do contain, cannot be considered harmless. 


\subsection{Toxicology}

Various chemical substances and ultrafine particles known to be toxic have been identified in e-cigarette aerosols, cartridges, refill liquids and environmental emissions:

- ultra-small aerosol particles - may increase the risk of cardiovascular disease;

- Nicotine - the highly addictive element of smoking;

- propylene glycol - a common and safe additive for food, but which has not been tested for ingestion into the lungs. There is some evidence of it being an airways irritant;

- diethylene glycol - linked to lung damage;

- flavourings - mostly thought to be harmless but cinnamon, vanilla and buttery flavours have been found to impact health;

- metals such as lead, nickel, manganese, chromium and cadmium;

- diacetyl - a chemical linked to bronchiolitis obliterans or "popcorn lung";

- acetaldehyde and formaldehyde - known carcinogens

- acrolein - has been shown to cause irreversible lung damage.

The majority of research is showing that these chemicals are present at lower levels in e-cigarette output than in cigarette output, although how much safer this makes them and whether they increase the risk of cancer, heart disease and lung problems, and by how much, is still a subject for further investigation.

\subsection{Impact on genetic material}

The basic cause of cancer is damage to DNA, causing mutations in genes which means cells multiply out of control and do not repair themselves properly. It is well established that mutations can be caused by tobacco smoke, which is why cigarettes have a proven link with cancer.

Chemicals known to damage DNA include formaldehyde, acrolein and methylglyoxal, all of which are present in e-cigarette vapour. Preliminary studies show that they are present in sufficient quantity to cause DNA changes. Research published by Lee et al. (2018) found that e-cigarette vapour exposure caused DNA damage in the lungs, bladder and heart of mice. Another small study carried out in 2018 by Balbo and Dator (2018) investigated the effect of a 15-minute vaping session on oral cells in five people and found that acrolein had reacted with DNA in their cells. In view of these results, a much larger study is now planned over a longer term.

The aim of future research will be to see whether cells can repair the damage and replicate normally, or whether they are going to continue down the path of malignant change.

\subsection{Tissue damage}

The majority of research about the health impacts of e-cigarettes is taken from epidemiological studies and clinical trials, but there have also been a number of laboratory-based investigations which test the cytotoxicity of chemicals in e-liquids, and those generated during aerolisation, using animal and human tissue. These investigations look at the effects of vaping on endothelial cell dysfunction and oxidative stress.

Vascular endothelium is the thin layer of cells lining the blood vessels. It has a complex role in actively controlling many functions. One function is to help regulate blood flow by relaxing the arterial walls and increasing blood flow, thereby lowering blood pressure which is important if the heart rate rises, for example, during exercise. Reduced vasodilation through stiffened arterial walls is associated with hypertension, coronary artery disease, peripheral vascular disease and heart and kidney failure. A healthy endothelium also suppresses thrombosis and vascular inflammation, both of which are associated with atherosclerosis and heart disease. It therefore follows that a dysfunctional endothelium leads to an increase in these conditions. 
The effect of tobacco smoke on endothelial cells, and the resulting increased cardiovascular risk, is well documented and accepted. The question is whether vaping has a similar effect.

There is substantial evidence that e-cigarettes can induce endothelial cell dysfunction.

Several studies have investigated the effect of e-cigarette aerosol extracts on human umbilical vein endothelial cells (HUVECs), looking at the response to exposure of different liquids and concentrations of nicotine. The effect was measured using the known responses to combustible tobacco, including nitric oxide net availability, reactive oxygen species (ROS) generation, expression of adhesion molecules, regeneration of endothelial cells, cell morphology and DNA damage.

Exposure to extracts from e-cigarette aerosols does provoke these responses in HUVECs but often to a lesser extent. An example of this is a study published by Anderson et al. (2016) which showed a 4.5-fold increase in ROS levels over control values. However, this value is lower than the 7.8-fold increase produced by combustible tobacco cigarette smoke.

Schweitzer et al. (2011) carried out two studies in 2011 comparing the effect of tobacco and e-cigarettes on pulmonary epithelium and endothelium cells of rat lungs and human bronchial cells. The effects of e-cigarette components were similar to that of combustible tobacco, but diminished.

Oxidation is the metabolic process of our cells. Oxidative stress is caused by the inability to detoxify natural metabolic compounds, leading to cell damage and inflammation. This causes a vast number of diseases including cancer and heart disease. Cigarette smoking is well known to cause oxidative stress, and current emerging research is showing that oxidative stress can also be caused by e-cigarette use, although at lower levels, and results have been mixed.

Studies carried out on bronchial epithelial cells by Anthérieu et al. (2017), kidneys in rats by Golli et al. (2016), and mouse lungs, Lee et al. (2018), did not show increased levels of oxidative stress when exposed to e-cigarette components. However, Ji et al. (2016) found an increase in oxidative stress on oral epithelial cells.

\subsection{Cardiovascular impact}

Cardiovascular events are rare prior to age 60. Given the immaturity of the e-cigarette market, and the fact that e-cigarette users are on average 7 years younger than smokers, it may take a significant period of time before e-cigarette usage can be demonstrated to result in actual cardiac events. This leaves the industry exposed to the long-tail risk which may yet emerge: a particular concern for long-term life cover and critical illness products.

While it is likely that e-cigarettes cause less harm to the cardiovascular system than smoking, we have already seen that research suggests they can alter the heart rate and blood pressure, which are precursors to vascular disease.

Several studies indicate that e-cigarette use elevates the risk of experiencing a stroke or myocardial infarction, and Bhatta \& Glantz (2019) even suggest that dual usage is riskier in this context than using either cigarettes or e-cigarettes alone. If so, given the promotion around using e-cigarettes as a cessation aid, this could have negative, unintended health repercussions.

Myocardial infarction is not the only cardiovascular risk that has been examined. Another study found there were no adverse effects on left ventricular myocardial function after use of an e-cigarette, unlike cigarettes, demonstrating that e-cigarettes could have a lower short-term cardiovascular risk compared to cigarettes (Farsalinos et al. 2014).

Ultimately, although there are indicators of increased cardiovascular risk due to e-cigarette usage, the majority of papers and experts agree that the risk is potentially lower than that of cigarette smoking. A recent study by Farsalinos et al. (2019), which uses the Centers for Disease Control and Prevention 2014, 2016 and the 2017 National Health Interview Survey data, found that even though e-cigarette users may have an increased risk of a heart attack by up to $55 \%$ 
compared to non-smokers, this compares to an increase of $165 \%$ for cigarette smokers. There was no statistical evidence to suggest that the risk of a stroke was any different for e-cigarette users, again in stark comparison to the $78 \%$ increased risk for smokers.

Other studies found evidence to contradict this. Prasad et al. (2017) looked at the increased risk of stroke and found that the levels of oxidative stress produced by e-cigarette aerosol and combustible tobacco smoke were the same in mouse brain cells, and the authors concluded from this and other tests that e-cigarette exposure is as damaging as that of combustible tobacco smoke. The evidence appears somewhat contradictory, so it is clear that further research is needed to study oxidative stress and the implications of that for e-cigarette safety.

\subsection{Respiratory function}

Cigarettes have long been known to affect the respiratory system. The interaction of the chemicals contained in tobacco with the lungs and respiratory system has been closely studied and understood for some time. The impact of how e-cigarettes and the chemicals they contain interact with the respiratory system is much less well known, and the issue is complicated by the sheer variety of flavourings and chemicals that can be found in e-cigarettes.

The chemicals contained in e-cigarettes have been shown to cause clear changes in lung biology, although it is not clear yet whether this results in lung disease (Husari et al., 2016). It has also been shown that e-cigarette vapour boosts the production of inflammatory chemicals and disables key protective cells in the lung (Allen et al., 2016).

E-cigarette vapour contains diacetyl, a chemical which causes a severe respiratory disease called bronchiolitis obliterans in microwave popcorn workers (and therefore commonly referred to as "popcorn lung") because in their working environment they inhale heated vapours containing the chemical. This has been widely reported in the media, mostly based on a Harvard study in December 2015 that found the presence of diacetyl in 39 of 51 flavours tested (Allen et al., 2016). The study did not confirm that the presence of diacetyl in vapour caused lung problems, but because of the association between diacetyl and bronchiolitis obliterans among workers inhaling heated vapours, it recommended urgent action to further evaluate the extent of this new exposure. The use of the chemical in e-liquids was banned in the UK in 2016 under the EU Tobacco Products Directive (TPD).

All the studies that have reported are on a small scale, and there have been no long-term studies completed. Although evidence suggests e-cigarettes can cause respiratory problems, until such long-term studies are undertaken the extent of the issue and how it compares with the problems caused by tobacco smoke is unknown.

\subsection{Cancer}

While e-cigarettes may not categorically cause cancer, we have already seen there is evidence that e-cigarettes may cause precursor events to cancer, such as lung inflammation and DNA damage.

The research carried out by Lee et al. (2018) is the first study to definitively link vaping with cancer. Interestingly, although nicotine has not been regarded as carcinogenic, in Lee's study $22.5 \%$ of the mice exposed to vapour with nicotine developed lung cancer during the 4-year trial, but no mice developed cancer if they were only exposed to e-cigarette vapour without nicotine. The researchers felt that it would be another decade before they had enough research to evaluate the impact of e-cigarettes on humans.

\subsection{Cessation}

Data on the impact of e-cigarettes on smoking cessation are very limited. Again, there are contradictory sources but few of these are studies of the form that would give us any comfort. One 
study by Hajek et al. (2019) produced in January 2019 looked at 886 National Health Service (NHS) stop smoking services users and randomly allocated them to two groups - one group given traditional nicotine replacement therapy (NRT) such as patches and one given e-cigarettes. After 1 year of follow-up, the proportion of those who were assigned e-cigarette who were still nonsmokers was $18 \%$ compared to $9.9 \%$ of those assigned other NRT products. This implies that e-cigarettes are twice as effective as traditional NRT in helping people quit but a more conservative view would be that even then $82 \%$ of people had relapsed back to smoking.

\section{Public Health England view}

Public Health England (PHE) is an executive agency of the Department of Health and Social Care, bringing together public health specialists from over 70 organisations to provide the government, local government, the NHS, parliament, industry and the public with evidence-based, professional scientific expertise and support. Its aim is to protect and improve the nation's health and wellbeing and reduce health inequalities. PHE works closely with public health professionals in Wales, Scotland and Northern Ireland.

PHE has been monitoring e-cigarettes and heated tobacco products and produced its first two reports in 2014 (PHE, 2014) and a third update in February 2019 (PHE, 2019). PHE used peerreviewed literature, surveys, reports and data sourced by, and made available to, a panel of leading independent tobacco experts.

PHE made the statement that e-cigarettes are 95\% less harmful than cigarettes, which drew criticism from some, not for stating that e-cigarettes are less harmful, but because of the figure of $95 \%$ which appears to be an estimate. As we have seen, the general consensus of opinion is that more research is needed before the safety of e-cigarettes can be verified.

PHE made the following points in their initial report.

- The long-term impact of nicotine on lung tissue is not yet known.

- Further research is needed on the similarities and differences in addictiveness of e-cigarettes and tobacco cigarettes and the potential harms associated with inhaled nicotine.

- E-cigarettes are not attracting many young people who have never smoked.

- The prevalence of e-cigarette use in adults in the UK has plateaued at $6 \%$ of the adult population.

- Never smokers are rarely taking up e-cigarettes.

- Socio-economic differences in e-cigarette use by smokers and recent ex-smokers have become smaller with no clear gradient in prevalence by occupational grade.

- The prevalence for dual use is similar for e-cigarette users and NRT users.

- Nicotine concentration is now limited by law to $20 \mathrm{mg} / \mathrm{ml}$.

- The most common reason for e-cigarette use continues to be to help stop smoking. However, the relapse rate is not yet known.

- More research is needed into patterns of e-cigarette use and its impact on health.

- There is currently no evidence that flavourings in e-cigarettes pose a health risk.

- $44 \%$ of smokers in the UK think e-cigarettes are equally or more harmful than smoking.

\section{Modelling}

There are many different types of e-cigarette users (which are not mutually exclusive, and include): 
- cessation aid smoker - users utilising e-cigarettes as a method of giving up smoking or reducing their current smoking intake;

- committed smoker - supplementing their existing smoking habits with additional e-cigarette smoking;

- e-cigarettes only/dual user - users solely smoking e-cigarettes or using them in conjunction with cigarettes;

- nicotine/non-nicotine user - users using e-cigarettes with or without nicotine;

- regular/social smoker - smoking e-cigarettes regularly (normally defined as at least one cigarette per day) or socially using e-cigarettes; and

- inhalation behaviour - number of puffs smoked and depth of vape inhalation.

Currently, the insurance industry applies a "one size fits all” approach to smokers with regard to pricing and underwriting. Given the huge variety of smoker types and habits, this is a rather crude, as well as an incorrect, way of pricing the relative risk as compared to non-smokers.

Unfortunately, the data/figures/statistics needed to accurately distinguish and therefore price between these smoker "types" is unavailable, and moreover, there are no reliable (and affordable) methods for insurance applicants to prove which category of smoker they fall into.

Also, in addition to the non-binary nature of smoking in the $21^{\text {st }}$ century, there is the fluidity of smoking statuses, where smokers are less likely to stick to just one category of smoker status for the duration of a long-term insurance contract. Therefore, we must also effectively price in the future transition rates between smoker states in order to "perfectly" price the risk.

In a traditional smoker/non-smoker world, the modelling is a relatively simple case of a threepart multi-state model with non-smokers (including ex-smokers and "never smokers"), smokers and dead.

Diagrammatically this looks as shown in Figure 2:

When we introduce e-cigarettes, we have a far more complex model (Figure 3 where the various transitions will depend on the relative weighting of various smoking categories):

When we consider that each of these transitions requires parameters that potentially vary by age, sex, period since quitting/starting, socio-economic group, level of smoking and so on, it becomes clear just how complex the modelling is. Currently, it is impossible to derive estimates of these parameters in a crude form, let alone anything remotely accurate. Therefore, instead of trying to offer an overly complex, but yet spuriously accurate model, we have done something essentially quite simple. We have looked at the impact of e-cigarettes on enhancing the quit rate and have assumed that the extra "quitters" stay with e-cigarettes and that their risk changes from smoking risk to e-cigarette risk over a fixed period.

There are currently 7.2 million smokers in the UK according to Action in Smoking and Health, (2019). If e-cigarettes did in fact enhance the quit rate, say by $2 \%$ per annum, and assuming that the ex-smoker mortality rate reverts to a non-smoker rate over a period of 15 years then, over the same period, extra quitters from e-cigarettes could lead to upwards of 7,500 deaths per year saved across the UK population.

The model works as follows:

- assuming a block of protection term assurance (scaled to current UK term in-force volumes)1

- all modelled with one single model point (20-year term, age 40 , male);

- modelled assuming that

- non-smokers are at $100 \%$ relative mortality;

- smokers are set with relative mortality as per TSM00/TNM00;

- e-cigarette users are all "recent quitters", and their mortality starts at that of smokers and reduces down linearly to that of non-smokers over a 15 -year period; 


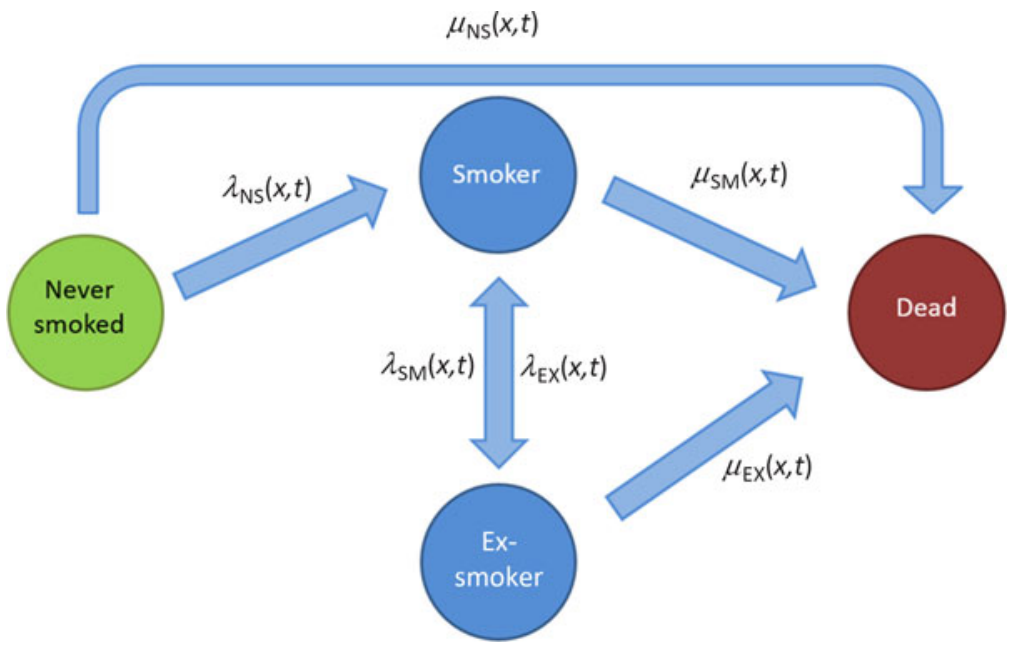

Figure 2. Multi-state model pre e-cigarettes.

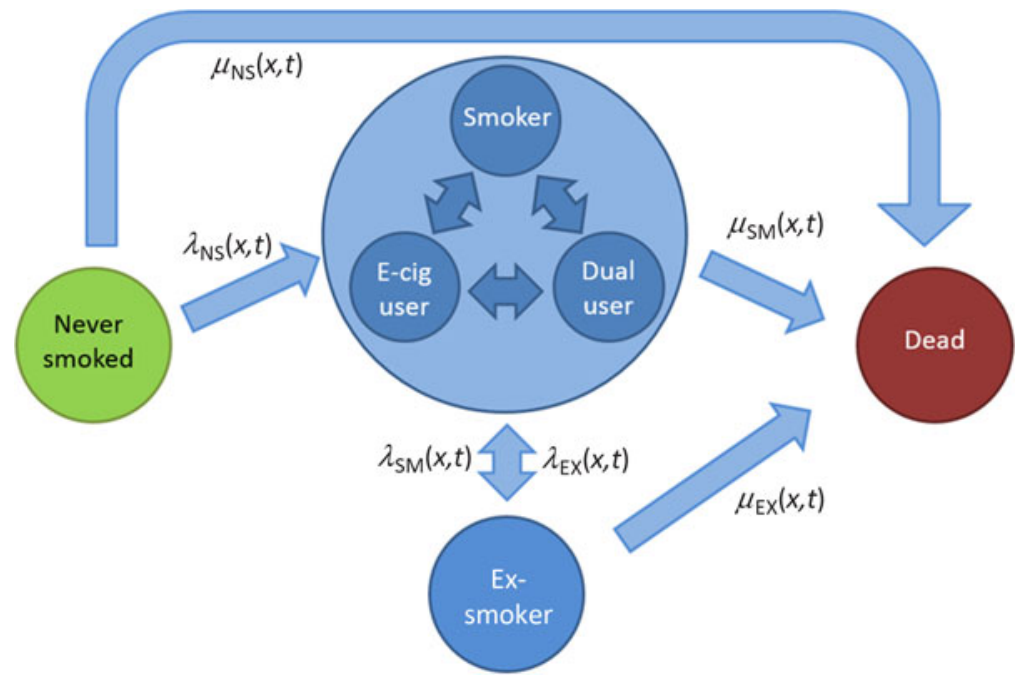

Figure 3. Multi-state model post-introduction of e-cigarettes.

- e-cigarettes lead to a higher smoking cessation rate (tested various scenarios);

- e-cigarettes are a certain \% safer than tobacco (tested scenarios on the \% safer);

- standard market approach to lapses and discount rates.

Using a total term market in force of $£ 4,400 \mathrm{~m}$, then additional smoking cessation could lead to an improved position for the UK term assurance portfolio as set out in Figure 4.

There are a few key simplifications and limitations to the model.

- Relapse rates. These have not been modelled but are not trivial. A recent study (Hajek, et al., 2019)shows that $82 \%$ of those who tried to quit relapsed back to smoking after 1 year. Isolating the relapse patterns for e-cigarettes is unfortunately not yet possible. It is also worth 


\begin{tabular}{|c|c|c|c|}
\hline Scenario & $\begin{array}{l}\text { E-cigarettes are } \\
\text { what \% safer } \\
\text { than cigarettes }\end{array}$ & $\begin{array}{l}\text { E-cigarettes result in an } \\
\text { increased smoking } \\
\text { cessation rate by what \% }\end{array}$ & $\begin{array}{l}\text { Total present value of claims - } \\
\text { Impact } \\
\text { (UK term assurance portfolio) }\end{array}$ \\
\hline $\begin{array}{l}\text { E-cigarettes are the same } \\
\text { risk as cigarettes, no } \\
\text { impact on smoking } \\
\text { cessation }\end{array}$ & $0 \%$ & 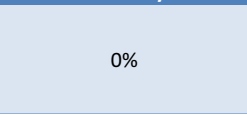 & . \\
\hline $\begin{array}{l}\text { PHE view on e-cigarette } \\
\text { relative risk, } 1 \% \text { increase } \\
\text { in smoking cessation }\end{array}$ & $95 \%$ & $1 \%$ & £458m \\
\hline $\begin{array}{l}\text { PHE view on e-cigarette } \\
\text { relative risk, } 2 \% \text { increase } \\
\text { in smoking cessation }\end{array}$ & $95 \%$ & $2 \%$ & $£ 863 \mathrm{~m}$ \\
\hline $\begin{array}{l}\text { E-cigarettes are } 50 \% \\
\text { safer than cigarettes, } 1 \% \\
\text { increase in smoking } \\
\text { cessation }\end{array}$ & $50 \%$ & $1 \%$ & $\mathrm{f} 241 \mathrm{~m}$ \\
\hline $\begin{array}{c}\text { E-cigarettes are } 10 \% \\
\text { safer than cigarettes, } 5 \% \\
\text { increase in smoking } \\
\text { cessation }\end{array}$ & $10 \%$ & $5 \%$ & £191m \\
\hline
\end{tabular}

Figure 4. The impact on UK term market value under various e-cigarette risk scenarios.

noting that further developments in the delivery of nicotine in e-cigarettes could potentially reduce relapse rates in the future.

- Gateway risk. The impact of non-smokers taking up e-cigarettes has not been modelled. This would have a negative impact on the scenarios modelled above; however, despite this being viewed as a material risk in the US, the view in the UK is that the risk is low.

- Dual usage. The impact of dual usage has not been taken into account. It has been assumed that the key use of e-cigarettes is in aiding smoking cessation, not complementing or replacing their current smoking habits.

As can be seen from Figure 4, the impact on the total claims value is highly dependent on the parameters chosen, and although very simplistic modelling, it does show that e-cigarettes could have a very significant positive impact on the value of protection books if these assumptions are borne out. The industry should continue to monitor emerging evidence as it becomes available, in order to develop the most accurate view of the impact of this emerging field.

\section{Survey of protection underwriters}

Two surveys of protection underwriters in the UK market have been conducted to better understand their thoughts and approach to e-cigarettes (The Institute of Actuaries: E-cigarette Working Party, 2017 and 2019). The recipients of the survey were largely the same group of people.

All respondents have an official position on e-cigarettes, with the majority treating users in the same manner as smokers. Previously, in 2017, a number of offices stated that they treat e-cigarettes users as non-smokers with a loading but there has been a shift to applying smoker rates for all ecigarette users, with one respondent stating, "this is based on Reinsurance changes and our own CMO view". This is despite acknowledgement of "increasing pressure to treat [e-cigarette users] differently to smokers".

Most application questions still do not differentiate between different types of tobacco usage, and there were only a handful of respondents who specifically include an e-cigarette question as part of the application process. Furthermore, fewer than half of those surveyed in both 2017 and 2019 actually collate and utilise the data collected on tobacco and/or e-cigarette usage.

There has been an increase since 2017 in the number of companies conducting research on ecigarettes. However, still fewer than a fifth are heading their own primary research (see Figure 5). 


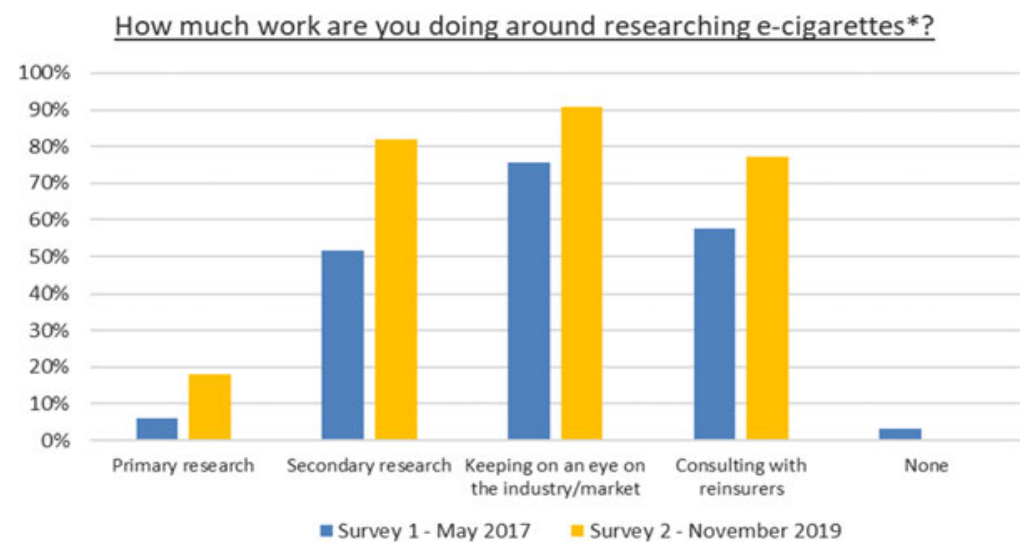

Figure 5. Survey question on the quantity of research being done by offices.

${ }^{*}$ Note - respondents may select multiple answers

Further published research and increased prevalence of e-cigarette smokers are considered the biggest drivers of an expected change in attitude towards e-cigarettes. However, changes in legislation (UK or global) are consistently not seen to be a significant driver.

There has been an increase in the number of respondents who expect there to be an industrywide change in attitude towards e-cigarettes, increasing from $82 \%$ to $95 \%$, with the majority of respondents now believing this will be seen within the next 3 years.

However, these surveys demonstrated that the direction of this expected change is highly debated.

\section{Product design}

The uncertainty around the actual risk of e-cigarettes and their impact on current and future health as well as their effect on smoking cessation rates makes it difficult to develop traditional long-term guaranteed products.

There is strong evidence (e.g. WHO (2019), NHS (2019) and Johns Hopkins Medicine (2019)) that the risks for ex-smokers follow a curve which is highest immediately following quitting and then drops steadily, almost to a level of a never smoker after a period of time. This period is often cited as 10-20 years, although other factors such as the period of smoking, the intensity of smoking and the specific medical condition in question will all influence the shape and duration of the reduction in relative risk. The impact of smoking cessation on life expectancy (Jha et al., 2013) can be seen in Figure 6.

Currently, insurance products are offered with rates on a binary basis: smoker versus nonsmoker. These could be replaced with a grading of rates from smoker to recent ex-smoker to a long-term ex-smoker and finally, a never smoker. See Figures $7 \mathrm{a}$ and $7 \mathrm{~b}$.

The shift away from binary smoking rates was in fact already starting to be developed prior to the launch of e-cigarettes. However, their emergence and following surge in popularity means an extra dimension to the smoker rating curve conundrum as to where an e-cigarette user may belong.

There is a variety of product features that could be considered to mitigate the uncertainty.

Reviewable contracts could be used to reduce the exposure to uncertainty over the relative risk of e-cigarettes over time. A certain assumption about their risk, for example, "e-cigarettes are $\mathrm{xx} \%$ safer than cigarettes", could be used and the pricing set accordingly, and reviews used if this assumption is not borne out in practice. However, on current evidence insurers possibly should 

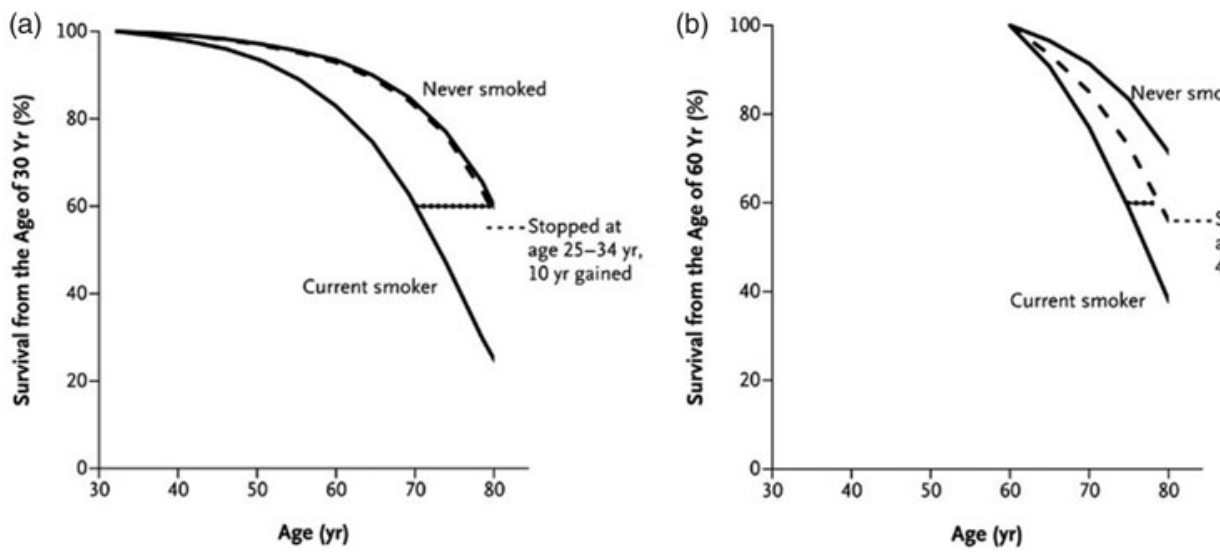

Figure 6. The impact of introducing e-cigarettes on risk profit. (a) Impact of smoking cessation on life expectancy of a 30 year old. (b) Impact of smoking cessation on life expectancy of a 60 year old.

(a)

\section{Current binary pricing} approach:

Non-smoker

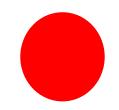

Smoker

(b)

\section{Possible non-binary pricing approach:}

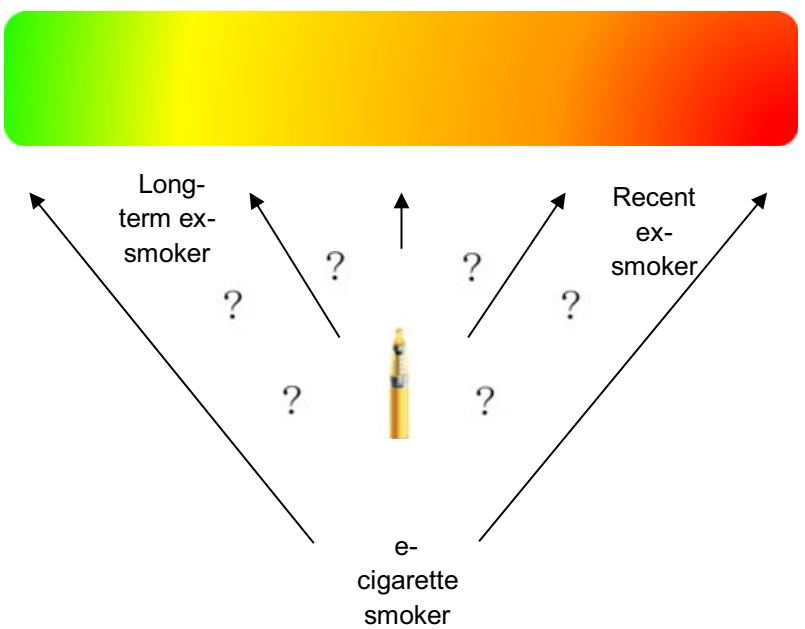

Figure 7. (a) Binary smoking model. (b) Realisation that smoking is not binary.

be hesitant about being too aggressive with the opening assumption. Conversely, if insurers are too conservative, it could be to the detriment of customers using e-cigarettes; they may end up paying too high a premium for their risk. Finally, the life industry is slowly moving away from reviewable contracts so we doubt that this will be a widely championed solution. 
Perhaps the motivation behind e-cigarettes usage should be taken into account. Those using e-cigarettes for the purposes of smoking cessation could be given a transitional rate whereby they then work towards a final end or "goal" rate when they finally become smoke-free. Ultimately, companies should be encouraging as many customers as possible to become smoke-free, and this is an opportunity to incentivise and influence behaviour. As per Office for National Statistics (2018) data, the main reasons in the UK given for using e-cigarettes by cigarette smokers and ex-smokers are as an aid to stop smoking (53.8\%), and the perception that they are less harmful than cigarettes $(10.6 \%)$. So, cigarette smokers are trying to make "health-conscious" decisions regarding their smoking.

Using e-cigarettes as a smoking cessation tool is different from:

- smokers who are migrating from cigarettes to e-cigarettes but have no intention of quitting;

- smokers who may be supplementing their traditional smoking habits with increased frequency, strength and/or volume of smoking; and

- people who have started smoking e-cigarettes despite never smoking cigarettes.

It is very difficult to distinguish, and consequently price, for these scenarios.

The contract reviewability aspect could also apply to the smoker status of the customer, rather than just the rates themselves. Customers could be asked to complete an annual assessment of their smoking status. An initial rate could be given based on their smoker status at that point in time. For example, if a customer was a current smoker, smoker rates would be applied. Each year following, they would be required to verify their smoker status, comparable to the Vitality (2019) approach to certain health factors. This could be via self-certification with randomised checks or could even utilise independent testing and verification if efficient and costeffective. The annual process could be streamlined by implementing certain rules. For example, lifelong non-smoker rates apply after five successive years of non-smoker certification. The administrative burden of this course of action, associated costs and inconvenience to the customer would have to be considered.

Similarly, short-term contracts may also mitigate some uncertainty around pricing for e-cigarettes by reducing the funnel of doubt. However, this does not address customers' need for long-term insurance.

The design of products and pricing is dependent upon on the ability to reliably assess an applicant's rating factors and associated risk. Insurers need to be confident that they could accurately ascertain smoking habits and, where applicable, the period since quitting for any form of smoking. The current cotinine test simply identifies the presence of nicotine by-products and is not helpful in discriminating between different sources of nicotine exposure. Tests are being developed but, before we can rely on them, we will need confidence in their accuracy.

Whilst none of the major insurance players in the UK have taken any considerable steps in the direction of a spectrum of smoker statuses, one of the most well-known tobacco companies, Philip Morris, has entered the insurance market with the launch of Reviti (2019). It is underwritten by Scottish Friendly Assurance Society. Reviti offers long-term term-life insurance with a number of different non-smoker and smoker statuses as shown in Figure 8.

Reviti offers a discount on premium rates to smokers who have switched from cigarettes to alternative smoking devices. The discount is up to $25 \%$ for those switching to IQOS (Philip Morris' own heat-not-burn product) but only $15 \%$ discount to those who switch to any other e-cigarette or vaping device. Whilst this is a step in the direction of recognising that smoking is not binary and need not be priced as such, the neutrality, evidence and motivation behind this product offering should be interrogated closely given that they are, in effect, encouraging purchases of IQOS devices as part of the insurance cover. As previously stated, the evidence to accurately determine the appropriate price differential (if any) and the short-term and long-term adherence to e-cigarettes over cigarettes is not readily available yet, let alone the ability to 


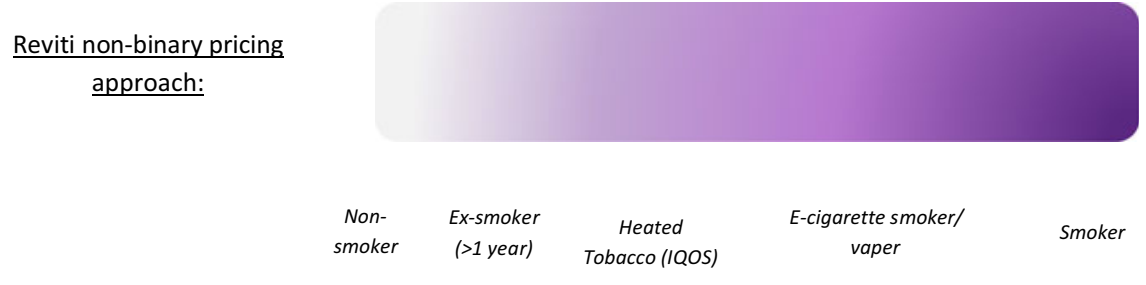

Figure 8. The potential risk profile of various smoking statuses.

determine the risk differential between various different types of e-cigarettes, heat-not-burn devices and alternative vaping products. Ultimately, it is debatable whether Phillip Morris International (2019), who have stated that their vision is "these products will one day replace cigarettes" should be spearheading the approach for the insurance industry.

Comparison and aggregator sites, underwriting manuals and industry platforms such as UnderwriteMe are possible industry focal points where a change may instigate a shift in the industry in a multitude of areas - advisers, underwriting, research, pricing and product design. However, on currently available evidence, such a change is unlikely. Data collection on smoking and behavioural understanding of customers must be prioritised in order to increase knowledge and understanding of e-cigarettes (and other alternative smoking devices) collectively within the industry. This is not currently the case as demonstrated in the surveys Institute \& Faculty of Actuaries (IFoA) (2017) and IFoA (2019). Despite almost 85\% of companies collecting information on e-cigarettes as part of the application process, fewer than half of those are actually collating or utilising this information.

\section{Legislation}

Legislation under the Health Act 2006, which prohibits smoking cigarettes in enclosed public places and workplaces, on public transport and in vehicles used for work, is based on conclusive scientific evidence of the direct health harm caused to bystanders through the inhalation of second-hand smoke. E-cigarette use is not covered by smoke-free legislation.

The TPD 2014/14/EU introduced new rules for nicotine-containing electronic cigarettes and refill containers from May 2016. New rules include

- maximum capacities and nicotine strength allowed

- tank capacity: $2 \mathrm{ml}$;

- e-liquid refill container capacity: $10 \mathrm{ml}$;

- nicotine strength of e-liquid: $20 \mathrm{mg} / \mathrm{ml}$;

- Other safety and quality standards:

- child-resistant and tamper evident packaging;

- prohibition of certain additives such as colourings;

- protection against breakage and leakage, and a mechanism for ensuring re-filling without leakage;

- Retailers and producers of e-cigarette products cannot be advertised or promoted, directly or indirectly:

$\circ$ on TV or on-demand TV;

on radio;

o through information society services (this includes, e.g. Internet advertising and commercial e-mail);

- in certain printed publications: newspapers; magazines; periodicals and similar publications; 
- the directive also prohibits

- sponsorship of television and radio programmes which promote electronic cigarettes;

- product placement of electronic cigarettes;

- sponsorship of activities or individuals that involve or take place in two or more European

Economic Area states or otherwise have cross border effects.

These restrictions are more limited than those which apply to tobacco advertising, where all forms of advertising and sponsorship by tobacco companies are prohibited. The 2015 prohibition on selling e-cigarettes to under-18s, and on adults buying them on behalf of under-18s, has also provided additional protection for children and young people.

\section{Underwriting and claims}

\subsection{Underwriting}

The basic premium for smokers is higher than that for non-smokers, so there has in the past been a high level of misrepresentation of smoking habits at application stage. This has resulted in a drop in premium income, which is compounded by a rise in claims expenses. When cotinine testing was introduced as a method of confirming whether someone was a non-smoker, the level of misrepresentation fell, largely due to the sentinel effect of testing. Today, even though only a small percentage of cotinine tests are positive, changing this proportion of applicants from non-smoker rates to smoker rates increases the annual premium enough to go some way to mitigate the effect of misrepresentation.

Cotinine is a chemical which is produced by the body after exposure to nicotine. It can be detected in the urine or blood and is regarded by insurers as a reliable test to confirm whether someone is a non-smoker. Stop smoking products such as patches, gum and liquids containing nicotine will also result in a positive cotinine test, and it is not possible to detect whether the cotinine is present as a result of cigarette smoking or the use of another product containing nicotine - including e-cigarettes. This is not of concern at the moment because anyone using NRT is treated as a smoker.

If a new tier of pricing is introduced, misrepresentation has to be considered again, and it will be difficult to prove whether someone is using e-cigarettes, tobacco or both, if cotinine tests cannot distinguish between them. If a customer tells us they are only using e-cigarettes and has a positive cotinine test, there is a risk they would be able to say it was due to e-cigarettes and obtain a cheaper premium rate, even if they smoke tobacco.

Cotinine testing is carried out by laboratories working with the insurance industry. They are currently working on a combination of test assays that will be able to distinguish between cigarette and e-cigarette use. This could include a combination of cotinine, $\mathrm{CO}$ and anabasine.

Exhaled CO is present in tobacco smoke but not in vapour; therefore, it is present in the expired air of smokers but not vapers. If this test is done in conjunction with a cotinine test, this can indicate whether someone is smoking cigarettes:

- - ve cotinine and - ve $\mathrm{CO}=$ non-smoker, non-vaper;

- +ve cotinine and +ve $\mathrm{CO}=$ smoker, possible vaper;

- + ve cotinine and - ve $\mathrm{CO}=$ possible vaper.

There are some issues with this method of testing though. Everyone has a small amount of CO in their breath, and if they live in a polluted area, it can be quite high (although not as high as it is for a smoker). The cut-off point which confirms that someone is a smoker is more than 10 parts per million (ppm) and for a non-smoker it is fewer than 5ppm. Between those levels, someone 
could possibly be smoking, or they could be exposed to a higher amount of pollution, so setting the cut-off point in this range means that potentially we could miss some smokers, or we could be classifying someone a smoker when they are not. In addition, cotinine is present in the urine for 4 days after exposure to nicotine, whereas CO is only present for 2-6 hours. This short detection window means that it is much easier to manipulate a negative $\mathrm{CO}$ result by abstaining from smoking for a few hours than it is to manipulate the cotinine result.

Anabasine and anatabine are alkaloids seen in tobacco leaves but not in nicotine. Testing for these could therefore be used as a tool to distinguish smokers from those using NRT where the cotinine result is positive. This has been studied in the US by the United Network for Organ Sharing (UNOS) because there is a strict rule that only non-smokers are eligible for organ transplant, and therefore tobacco screening assays are a vital part of determining whether patients can be registered as a candidate as an organ recipient or not. If candidates are refused based only on their cotinine result, those on NRT would not be eligible for a life-saving organ, so laboratories have been trying to develop tests to assess whether a patient is smoking cigarettes or using (and is compliant with) NRT. For this to be reliable, these alkaloids have to be positive in smokers all of the time, but studies (e.g. Payton et al., 2002) have shown this not to be the case. Clinical information from UNOS showed that of 51 self-admitted smokers, 30 had negative anabasine tests. This means that if anabasine had been used to classify potential transplant patients, $60 \%$ could have been incorrectly classified as non-smokers, which would have a dramatic effect on the allocation of organs and outcomes for patients (Feldhammer \& Ritchie, 2017). A 60\% failure rate also indicates it would not be a reliable test for the insurance industry to use.

Laboratories in the UK are currently investigating ways in which a combination of tests can be used to differentiate reliably between cigarettes and e-cigarette use only, but these are not available yet.

Finally, users of conventional NRT are charged as smokers in view of the possibility of relapsing to smoking tobacco. Before we treat e-cigarette use differently, it has to be established that vaping is an effective long-term solution to stopping smoking, and there is no information available at present to confirm this.

\subsection{Claims}

Cancer and cardiovascular disease, both strongly linked to smoking, are conditions which account for a large proportion of both life and critical illness claims. If studies are correct and e-cigarettes are proved to be a less harmful option than cigarettes then, as an industry, we should start to see an improvement in claims experience.

Although, as an industry, we pay over $98 \%$ of claims across all products, based on claims data (Association of British Insurers, 2018), there is a small number of claims that are not paid due to misrepresentation. Smoking is one of the main areas of misrepresentation that claims assessors have to deal with. If a customer has misrepresented their smoking habits, it is usually discovered through a tele-claim call with the customer or through medical records that are obtained from a General Practitioner or treating consultant to assist in the assessment of a claim. As smoking history is provided in the medical records, it is usually hard for a customer to dispute and we have a clear audit trail of dates. Misrepresentation of smoking habits can lead to a reduction in the claim value that is actually paid and, in some circumstances, can result in no claim being payable at all. Currently, the process is clear, a customer can either be on smoker or non-smoker rates.

If an additional level of pricing is added in for e-cigarette users, there are two main areas of concern from a claims perspective:

1. The investigations into misrepresentation of smoking will become more complex. If a customer has disclosed themselves as an e-cigarette user only at application stage, in the event 
of a claim it may be very difficult for the assessor to prove whether this was in fact correct. At this time, we do not know how e-cigarette use will be recorded in medical records. It is well known that many e-cigarette users are dual users, but we will be reliant solely on what the customer tells us, so if the customer does not disclose this we will be totally unaware of it. If there is an element of doubt around smoking habits, then the claims decision will have to be made in favour of the customer.

2. Although customers may be genuine e-cigarette users at application stage, the relapse risk to cigarettes, and how that compares to those who have quit nicotine products completely, remains unknown currently. Any increase in relapse risk may lead to a higher proportion of smoking-related claims in the e-cigarette user risk pool than has been allowed for. The customer will not have been paying the full smoker premium rate, as there is currently no requirement for customers to advise an insurer if they become cigarette smokers again.

Both concerns will ultimately mean that there will be a number of claims paid where the customer has not paid the correct premium for the risk presented, which would affect the industry's claims' experience. However, it is hoped that this negative impact will not outweigh the positive impact of health improvements.

\section{Recent media coverage}

In 2019, there was an increase in negative news regarding e-cigarettes circulating in the media.

Within the USA, recent reports have linked a number of deaths to the use of e-cigarettes. While this is a major concern, it is becoming more apparent that these deaths are linked to the consumption of unregulated liquids containing tetrahydrocannabinol (THC), the psychoactive element found in cannabis. It should be noted that THC is banned in the UK. While not all the recent US deaths have been linked with THC liquid, there are issues of non-disclosure as the consumption of cannabis is still illegal in several states.

A recent case in the UK where a teenager was in critical condition following usage of e-cigarettes has raised many questions around their safety. There is evidence that the medical distress was due to a severe allergic reaction to a component in the liquid. Despite this, UK public health experts still back the use of e-cigarettes as an alternative to smoking due to the rare and isolated nature of this case.

Again this demonstrates that long-term health risks remain unknown and further studies are required.

\section{Next steps}

Evidence is slowly emerging on e-cigarette safety and their effectiveness in helping people cease smoking completely. But it is fair to say that we are still some way from a clear, unambiguous view.

For a firmer view to be formed on e-cigarettes and their use and risk, insurance providers and the IFoA (through the Continuous Mortality Investigation (CMI)) could examine the feasibility of collecting mortality data which can then be dissected to a granular level beyond smoker/nonsmoker. Such a study would take time, first for the mortality data to emerge, and then to collect and collate it.

\section{Conclusions}

Smoking has had one of the most significant impacts on our industry in recent memory. It has had a major effect on mortality with a consequent need to amend pricing, reserving, underwriting and claims. But with time, the industry learned how to amend these various functions for the impact of 
smoking. E-cigarettes are still a relatively new phenomenon but also have the potential to have a very significant impact. The industry is yet to form a coherent view on how to allow for their impact.

We have considered a large number of sources of information but have found that some of it is quite contradictory. There is certainly not a unanimously accepted view on either the relative risk of e-cigarettes or their impact on quit rates. Nor are there any mortality studies yet. Hence, we have had to look at a number of other types of data which are proxies for possible mortality risk.

We simply cannot form a cast iron view that says "e-cigarettes are $\mathrm{xx} \%$ safer than smoking" with any certainty, and we feel that any attempt to write such a conclusion (even if suitably caveated) could be viewed as an official position of the IFoA. We do not feel that is helpful. However, we are of the opinion that the majority of evidence is pointing to a conclusion that e-cigarettes are safer than tobacco but not as safe as not smoking at all.

Our paper still has a number of important takeaways even without being able to offer such a conclusion. We also feel that this is a perfect time for our industry to put forward a coherent strategy as to how we will form these conclusions in a more robust way. We feel that the time is right for a body such as the IFoA, through the CMI, to examine the feasibility of collecting the necessary data that would allow us to better analyse the experience that is emerging. At the same time, the industry should continue to engage with the various experts in this expanding field in order to start to form a consensus that could become our best view before "real data" become available.

\section{Disclaimer}

The views expressed in this publication are those of invited contributors and not necessarily those of the Institute and Faculty of Actuaries. The Institute and Faculty of Actuaries does not endorse any of the views stated, nor any claims or representations made in this publication and accepts no responsibility or liability to any person for loss or damage suffered as a consequence of their placing reliance upon any view, claim or representation made in this publication. The information and expressions of opinion contained in this publication are not intended to be a comprehensive study, nor to provide actuarial advice or advice of any nature and should not be treated as a substitute for specific advice concerning individual situations. On no account may any part of this publication be reproduced without the written permission of the Institute and Faculty of Actuaries.

\section{References}

Action in Smoking and Health (ASH) (2019). In 2019 around half as many Britons now vape as smoke, and the majority are ex-smokers. Available at: https://ash.org.uk/media-and-news/press-releases-media-and-news/in-2019-around-half-asmany-britons-now-vape-as-smoke-and-the-majority-are-ex-smokers/ (Accessed 6 November 2019).

Allen, J.G., Flanigan, S.S., LeBlanc, M., Vallarino, J., Macnaughton, P. \& Stewart, J. (2016). Flavoring Chemicals in E-Cigarettes; Diacetyl, 2.3-Pentanedione, and Acetoin in a Sample of 51 Products, Including Fruit- Candy- and Cocktail-Flavored E-Cigarettes' Environmental Health Perspectives, 124(6), 733-739. DOI: 10.1289/ehp.1510185.

American Lung Association (2019). What's In a Cigarette? Available at: https://www.lung.org/stop-smoking/smoking-facts/ whats-in-a-cigarette.html (Accessed 6 November 2019).

Anderson, C., Majeste, A., Hanus, J. \& Wang, S. (2016). 'E-cigarette aerosol exposure induces reactive oxygen species, DNA damage, and cell death in vascular endothelial cells'. Toxicological Sciences, 154(2), 332-340. DOI: 10.1093/toxsci/kfw166.

Anthérieu, S., Garat, A., Beauval, N., Soyez, M. et al. (2017). 'Comparison of cellular and transcriptomic effects between electronic cigarette vapor and cigarette smoke in human bronchial epithelial cells', Toxicology in Vitro, 45(Part 3), 417-425. DOI: 10.1016/j.tiv.2016.12.015.

Association of British Insurers (ABI) (2018). UK Insurance and Long-Term Savings Key Facts. Available at: https://www.abi. org.uk/globalassets/files/publications/public/key-facts/key_facts_2018.pdf (Accessed 6 November 2019).

Balbo, S. \& Dator, R. (2018). Integrating multi-'omics'-mass-spectrometry-based methods to characterize electronic cigarette exposure in humans. Presented at ACS 256 $6^{\text {th }}$ National Meeting \& Exposition 2018. 
Bhatta, D.N. \& Glantz, S.A. (2019). 'Electronic Cigarette Use and Myocardial Infarction Among Adults in the US Population Assessment of Tobacco and Health', Journal of the American Heart Association. DOI: 10.1161/JAHA.119.012317.

Farsalinos, K.E., Polosa, R., Cibella, F. \& Niaura, R. (2019). 'Impact on Cardiovascular outcomes among e-cigarette users: A review from national health interview surveys', Journal of the American College of Cardiology, 73(9) Supplement 2: 11. DOI: 10.1016/S0735-1097(19)33773-8.

Farsalinos, K.E., Tsiapras, D., Kyrzopoulos, S., Savvopoulou, M. \& Voudris, V. (2014). 'Acute effects of using an electronic nicotine-delivery device (electronic cigarette) on myocardial function: comparison with the effects of regular cigarettes', BMC Cardiovascular Disorders, 14, 78. DOI: 10.1186/1471-2261-14-78.

Feldhammer, M. \& Ritchie, J.C. (2017). 'Anabasine is a Poor Marker for Determining Smoking Status of Transplant Patients', Clinical Chemistry, 63(2), 604-606. DOI: 10.1373/clinchem.2016.265546.

Golli, N.E., Jrad-Lamine, A., Neffati, H., Dkhili, H., Rahali, D., Dallagi, Y., May, M.V.E., Fazaa, S.E. (2016). Impact of e-cigarette refill liquid exposure on rat kidney', Regulatory Toxicology and Pharmacology, 77, 109-116. DOI: 10.1016/j. yrtph.2016.02.012.

Hajek, P., Phillips-Waller, A., Przulj, D., Pesola, F., Smith, K.M., Bisal, N., Li, J., Parrott, S., Sasieni, P., Dawkins, L., Ross, L., Goniewicz, M., Wu, Q. \& McRobbie, H.J. (2019). 'A randomised trial of e-cigarettes versus nicotine replacement therapy', New England Journal of Medicine, 380(7), 629-637. DOI: 10.1056/NEJMoa1808779.

Husari, A., Shihadeh, A., Talih, S., Hashem, Y., El-Sabban, M. \& Zaatari, G. (2016). 'Acute exposure to electronic and combustible cigarette aerosols: Effects in an animal model and in human alveolar cells', Nicotine \& Tobacco Research, 18(5), 613-619. DOI: 10.1093/ntr/ntv169.

Jha, P., Ramasundarahettige, C., Landsman, V., Rostron, B., Thun, M., Anderson, R.N., McAfee, T. \& Peto, R. (2013). '21st Century Hazards of Smoking and Benefits of Cessation in the United States', New England Journal of Medicine, 368, 341-350. DOI: 10.1056/NEJMsa1211128.

Ji, E.H., Sun, B., Zhao, T., Shu, S., Chang, C.H., Messadi, D., Xia, T., Zhu, Y. \& Hu, S. (2016). 'Characterization of Electronic Cigarette Aerosol and Its Induction of Oxidative Stress Response in Oral Keratinocytes', PLoS One, doi: 10. 1371/journal.pone.0154447.

Johns Hopkins Medicine (2019). Former Smokers: What's Your Risk for Lung Cancer? Available at: https://www. hopkinsmedicine.org/health/conditions-and-diseases/lung-cancer/former-smoker-whats-your-risk-for-lung-cancer (Accessed 6 November 2019).

Lee, H., Park, S. H., Weng, M. W., Wang, H.-T., Huang, W.C., Lepor, H., Wu, X.-R., Chen, L.-C. and Tang, M.-s. (2018). E-cigarette damages DNA and reduced repair activity in mouse lung, heart, and bladder as well as in human bladder cells', PNAS, 115(7), E1560-E1569. doi: 10.1073/pnas.1718185115.

NHS (2019). Smoke Free. Available at: https://www.nhs.uk/smokefree/why-quit/what-happens-when-you-quit (Accessed 6 November 2019).

Office for National Statistics (2018). Adult Smoking Habits in the UK, Available at: https://www.ons.gov.uk/ peoplepopulationandcommunity/healthandsocialcare/healthandlifeexpectancies/bulletins/adultsmokinghabitsingreatbr itain/2018 (Accessed 6 November 2019).

Payton, J., Hatsukami, D., Severson, H., Hall, S., Yu, L. \& Benowitz, N.L. (2002). 'Anabasine and Anatabine as Biomarkers for Tobacco Use during Nicotine Replacement Therapy', Cancer Epidemiology, Biomarkers and Prevention, 11(12), 16681673. Available at: https://cebp.aacrjournals.org/content/11/12/1668 (Accessed: 6 November 2019).

Phillip Morris International (2019). Delivering a Smoke-Free Future Available at: https://www.pmi.com/ourtransformation/delivering-a-smoke-free-future (Accessed 6 March 2020).

Prasad, S., Sajja, R. K., Kaisar, M. A., Park, J. H., Villalba, H., Liles, T., Abbruscato, T. \& Cucullo, L. (2017). 'Role of Nrf2 and protective effects of metformin against tobacco smoke-induced cerebrovascular toxicity', Redox Biology, 12, pp. 58-69. doi: 10.1016/j.redox.2017.02.007.

Public Health England (2014). Electronic Cigarettes. Available at:https://assets.publishing.service.gov.uk/government/ uploads/system/uploads/attachment_data/file/311887/Ecigarettes_report.pdf (Accessed 6 November 2019).

Public Health England (2019). Vaping in England: evidence update summary. Available at: https://www.gov.uk/government/ publications/vaping-in-england-an-evidence-update-february-2019/vaping-in-england-evidence-update-summaryfebruary-2019 (Accessed 6 November 2019).

Reviti (2019). Up first? Helping you to quit smoking. Available at: https://www.revitilife.com/ (Accessed 6 March 2020).

Schweitzer, K.S., Hatoum, H., Brown, M.B., Gupta, M., Justice, M.J., Beteck, B., Van Demark, M., Gu, Y., Presson, R.G., Hubbard, W.C. \& Petrache, I. (2011). Mechanisms of lung endothelial barrier disruption induced by cigarette smoke: Role of oxidative stress and ceramides', American Journal of Physiology — Lung Cellular and Molecular Physiology, 301(6), L836L846. doi: 10.1152/ajplung.00385.2010.

The Institute of Actuaries: E-cigarette Working Party (2017). Industry Underwriter Survey, Collection of Information at Application, IFoA, London.

The Institute of Actuaries: E-cigarette Working Party (2019). Industry Underwriter Survey, Collection of Information at Application, IFoA, London. 
Vitality (2019). Vitality Wellness Optimiser. Available at: https://www.vitality.co.uk/life-insurance/optimiser/ (Accessed: 6 November 2019).

Wikipedia (2019). Hon Lik. Available at: https://en.wikipedia.org/wiki/Hon_Lik (Accessed: 6 November 2019).

World Health Organisation (2019). Tobacco Free Initiative (TFI). Available at: https://www.who.int/tobacco/quitting/ benefits/en/ (Accessed: 6 November 2019).

Cite this article: Daniels N, Cosma C, Llewellyn A, Banks D, Morris H, Copeland J, and Djarlijeva E. E-cigarettes: no smoke without fire? British Actuarial Journal. https://doi.org/10.1017/S1357321720000112 